\title{
DIMENSION IN RINGS WITH SOLVABLE ALGEBRAIC GROUP ACTION
}

\author{
ANDY R. MAGID
}

In [3], Fossum and Foxby have shown that a number of properties of a graded commutative ring and its category of modules can be deduced from the corresponding properties for graded ideals and graded modules. If the Zgraded ring $R=\oplus R_{i}$ is an algebra over the field $k$, then the algebraic group $G$ $=\mathrm{GL}_{1}(k)$ can be made to act on $R: t$ in $G$ acts on $R_{i}$ by multiplication by $t^{i}$, and then a graded $R$-module is a simultaneous $R$ and $G$-module. This suggests that for a ring $R$ on which an algebraic group $G$ acts, various properties of $R$ can be deduced from the corresponding properties for simultaneous $R$ and $G$ modules. We show in this paper that this is indeed the case when the algebraic group $G$ is linear and solvable.

We fix the following notation: $k$ is an algebraically closed field and $G$ is a solvable linear algebraic group over $k$. A finite dimensional vector space $V$ with $G$-action is a $G$-module if the induced homomorphism $G \rightarrow \mathrm{GL}(V)$ is a homomorphism of algebraic groups over $k$. A vector space $W$ with $G$-action is a rational $G$-module if $W$ is a union of finite dimensional $G$-modules in the above sense. $\mathrm{A}$ *ring is a commutative Noetherian $k$-algebra which is a rational $G$-module such that $G$ acts by $k$-algebra automorphisms. A *module $M$ over a *ring $R$ is an $R$-module and a rational $G$-module such that $g(r m)=g(r) g(m)$ for $g$ in $G, r$ in $R$, and $m$ in $M$. A *homomorphism of *modules over a *ring $R$ is an $R$-module homomorphism preserving $G$-actions. A semi-invariant of weight $X$ in a rational $G$-module $V$ is a non-zero vector $v$ in $V$ such that $g(v)=X(g) v$ for some algebraic character $X: G \rightarrow \mathrm{GL}_{1}(k)$ of $G$. Since $G$ is solvable, every non-zero rational $G$-module contains a semi-invariant. An $*$ ideal of a *ring $R$ is an ideal which is a sub-*module. If $P$ is a prime *ideal of the *ring $R,{ }^{*}$ ht $(P)$ is the length of the longest saturated chain of prime *ideals ending with $P$, and ${ }^{*} \operatorname{dim} R$ is the supremum of ${ }^{*}$ ht $(P)$ as $P$ varies over prime *ideals of $R$. We call a *ring $R *$ simple if the only *ideals of $R$ are 0 and $R$. If $R$ is a *ring and $S$ is a multiplicatively closed set of semi-invariants in $R$, then $S^{-1} R$ is a ${ }^{*}$ ring and a *module over $R$.

In these notations, the main results may be summarized as follows: our

\footnotetext{
Received June 18, 1979.
} 
principal technical tool is the fact (Theorem 3) that a *simple *ring is regular of dimension at most $\operatorname{dim} G$. This implies that the Krull dimension of $R, \operatorname{dim} R$, is bounded by $* \operatorname{dim} R+\operatorname{dim} G$ (Theorem 7 ), and that projective and injective dimension of a *module can be bounded by considering Ext's just ranging over ${ }^{*}$ modules (Theorem 8 and Corollary 11 ). Finally, we show that a ${ }^{*}$ ring $R$ is regular (respectively Gorenstein) if all its localizations at *prime ideals are (Corollaries 12 and 13).

We restrict attention only to solvable groups to guarantee that a non-zero *ideal in a *ring contains a non-zero principal *ideal, which is necessary for induction arguments. Our solvable groups are not necessarily reductive, however, so semi-invariants in a *homomorphic image may not have semiinvariant preimages. It is this fact which prevents a good notion of "*localization", which means that a number of the techniques of [3] cannot be extended to our setting.

Lemma 1. Let $R$ be $a^{*}$ ring.

a) $R$ is * simple if and only if every semi-invariant is a unit.

b) If $R$ is a domain and $S$ is the set of semi-invariants of $R$ then $S^{-1} R$ is *simple.

Proof. Part a) follows immediately from the fact that non-zero $*_{\text {ideals }}$ contain non-zero principal *ideals. Then to establish $b$ ), we need to show that every semi-invariant of $S^{-1} R$ is a unit. So let $f / g$ in $S^{-1} R$ be semi-invariant of weight $\alpha$, where $f \in R$ and $g \in S$, with $g$ of weight $\beta$. For $t$ in $G$,

$$
\alpha(t) f / g=t(f / g)=t(f) / t(g)=t(f) / \beta(f) g,
$$

so $t(f)=\left(\alpha \beta^{-1}\right)(t) f$ and $f$ is semi-invariant, so $f / g$ is a unit in $S^{-1} R$.

Lemma 2. Let $R$ be a*ring which is an affine domain and let $S$ be the set of semi-invariants of $R$. Then $S^{-1} R$ is a regular ring of dimension at most the dimension of $G$.

Proof. We first bound the dimension of $S^{-1} R$. We can regard $R$ as the coordinate ring of an affine variety $V$ with $G$-action. Let $Q$ be a prime of $S^{-1} R$ and let $P=Q \cap R$. Then ht $(Q)=$ ht $(P)$ and $P \cap S=\varnothing$. The zero set $W$ of $P$ in $V$ is a closed subvariety with ht $(P)=\operatorname{codim}(W)$. Let $I=\{f \in R \mid f(G \cdot W)=0\}$. Then $I$ is an *ideal of $R$ contained in $P$. If $I \neq 0$, then $I$ contains an element of $S$. Since $P \cap S=\varnothing, I=0$, so $G \cdot W$ is dense in $V$. Then $G \cdot W$ contains a non-empty open subset $U$ of $V$. We may assume $U$ is $G$-stable. Moreover, $V$ contains a $G$ stable open subset $U^{\prime}$ such that a geometric quotient $U^{\prime} / G$ exists [6]. Let $U_{0}$ $=U \cap U^{\prime}$ and let $U_{1}=U_{0} \cap W . U_{1}$ is open in $W$ and closed in $U_{0}$, and $U_{0}$ 
$=G \cdot U_{1}$. Moreover, $\operatorname{codim}_{U_{0}} U_{1}=\operatorname{codim}_{V} W$, so we may assume that $G \cdot W=V$ and that the geometric quotient $V / G$ exists. Let $p: V \rightarrow V / G$ be the quotient map. The fibres of $p$ are orbits and hence have dimension at most $\operatorname{dim}(G)$, so $\operatorname{dim}(V / G) \geqq \operatorname{dim} V-\operatorname{dim} G$. Also, $p: W \rightarrow V / G$ is onto, so $\operatorname{dim} W \geqq \operatorname{dim}(V / G)$, and hence

$$
\operatorname{dim} G \geqq \operatorname{dim} V-\operatorname{dim} W=\operatorname{codim}_{V}(W)=\text { ht }(P) .
$$

To see that $S^{-1} R$ is regular, we note that since $S^{-1} R$ is a localization of an affine algebra, the singular locus is closed in $\operatorname{Spec}\left(S^{-1} R\right)$ [5, Thm. 73, p. 247]. Since the singular locus is $G$-stable, its defining radical ideal is an *ideal. By Lemma $1(\mathrm{~b}), S^{-1} R$ is *simple, so the singular locus is empty.

TheOREM 3. A simple * ring is a regular integral domain of dimension at most the dimension of $G$.

Proof. Let $R$ be a simple *ring. The minimal primes of $R$ are *ideals and hence $R$ is a domain. Write $R=\operatorname{dir} \lim R_{i}$, where $R_{i}$ are sub-*rings of $R$ which are affine, and let $S_{i}$ be the set of semi-invariants of $R_{i}$. By Lemma 1(a), $S_{i}^{-1} R_{i} \subseteq R$ so $R=\operatorname{dir} \lim S_{i}^{-1} R_{i}$. Let $T_{i}=S_{i}^{-1} R_{i}$. Suppose $T_{i} \subseteq T_{j}$, and let $U$ $=\left\{P \in \operatorname{Spec}\left(T_{i}\right) \mid\left(T_{j}\right)_{P}\right.$ is $T_{i}-$ flat $\}$. Then $U$ is open and non-empty in $\operatorname{Spec}\left(T_{i}\right)$ by generic flatness [5, Lemma 1, p. 156] and $U$ is clearly $G$-stable. Then the defining radical ideal of the complement of $U$ is an *ideal; since $T_{i}$ is *simple by Lemma 1 (b), the complement is empty and $T_{j}$ is $T_{i}$-flat. Since $R$ $=\operatorname{dir} \lim T_{i}$, and the inclusions $T_{i} \subseteq T_{j}$ are flat morphisms, $R$ is regular of dimension at most $\operatorname{dim} G$ since each $T_{i}$ is.

We now turn to computations of heights of prime ideals in a *ring. We will use the following construction:

Lemma 4. Let $P$ be a prime ideal of the ${ }^{*}$ ring $R$. The sum ${ }^{*} P$ of all the ${ }^{*}$ ideals contained in $P$ is a prime *ideal.

Proof. $P$ contains a minimal prime $P_{0}$ over ${ }^{*} P . P_{0}$ is necessarily an $*$ ideal so $P_{0}={ }^{*} P$ and hence ${ }^{*} P$ is prime.

Proposition 5. Let $R$ be $a{ }^{*}$ ring and let $P$ be a prime ideal of $R$

a) Some ranking chain of $P$ contains ${ }^{*} P$.

b) If $P={ }^{*} P$, ht $(P)={ }^{*}$ ht $(P)$.

Proof. We prove both parts simultaneously by induction on $n=$ ht $(P)$. If $n$ $=0$, then $P={ }^{*} P$ and both follow. To apply induction, we observe that if $I$ is an 
*ideal contained in $P$, then, by Lemma $4, I \subseteq{ }^{*} P$ and $\left({ }^{*} P\right) / I={ }^{*}(P / I)$. Now let $P_{0} \subseteq P_{1} \subseteq \ldots \subseteq P_{n}=P$ be a saturated chain of length $n=$ ht $(P)>0$. Then $P_{0}$ is a minimal prime of $R$ and hence an *ideal. Moreover, in $R / P_{0}$, ht $\left(P / P_{0}\right)=$ ht $(P)$. If a) and b) hold for $P / P_{0}$ in $R / P_{0}$, we can pull back the relevant primes to see that a) and b) hold for $P$ in $R$. Thus we may assume $R$ is a domain. If $* P=0$, a) holds. If not, $P$ contains a semi-invariant $x$. Let $I=R x$. $I$ is an $*$ ideal, and in the ring $R / I$, ht $(P / I)=n-1\left[4\right.$, Thm. 155, p. 113]. Let $Q_{1} \subseteq Q_{2} \subseteq \ldots \subseteq Q_{n}=P / I$ be a ranking chain for $P / I$ in $R / I$. By induction for part a), some $Q_{j}={ }^{*}(P / I)$. If $Q_{i}^{\prime}$ is the inverse image of $Q_{i}$ in $R$, then $0 \subseteq Q_{1}^{\prime} \subseteq \ldots \subseteq Q_{n}^{\prime}=P$ is a ranking chain for $P$ in $R$, and $Q_{j}^{\prime}={ }^{*} P$. By induction for part b), we may assume each $Q_{i}$ is an $*_{\text {ideal. With }} Q_{i}^{\prime}$ as inverse images, then, $0 \leqq Q_{1}^{\prime} \subseteq \ldots \leqq Q_{n}^{\prime}=P$ is a ranking chain for $P$ composed of $*$ ideals. Thus ht $(P)={ }^{*}$ ht $(P)$.

Corollary 6. Let $R$ be $a{ }^{*}$ ring and let $P$ be a prime ideal of $R$. Then ht $(P)$ $={ }^{*}$ ht $\left({ }^{*} P\right)+$ ht $\left(P /{ }^{*} P\right)$.

Proof. Let $n=$ ht $(P)$ and let $P_{0} \subseteq P_{1} \subseteq \ldots \subseteq P_{n}=P$ be a ranking chain for $P$. For each $i$, we have ht $(P)=\mathrm{ht}\left(P_{i}\right)+$ ht $\left(P / P_{i}\right)$. By Proposition 5 a), ${ }^{*} P=P_{i}$ for some $i$, and by Proposition $5 \mathrm{~b}), \mathrm{ht}\left({ }^{*} P\right)={ }^{*}$ ht $\left({ }^{*} P\right)$.

Using Theorem 3, we can bound the term ht $\left(P / P^{*}\right)$ of Corollary 6 , and hence determine the relation between dimension and *dimension for a *ring.

Theorem 7. Let $R$ be $a{ }^{*}$ ring and $P$ a prime ideal of $R$.

a) ht $(P) \leqq *$ ht $\left({ }^{*} P\right)+\operatorname{dim} G$.

b) $\operatorname{dim} R \leqq * \operatorname{dim} R+\operatorname{dim} G$.

c) ht $(P)={ }^{*}$ ht $\left({ }^{*} P\right)+n$, where $n$ is the minimal number of generators of $P R_{P} /{ }^{*} P R_{P}$ in $R_{P} /{ }^{*} P R_{P}$.

Proof. Let $\bar{R}=R /{ }^{*} P$, let $\bar{S}$ be the set of semi-invariants in $\bar{R}$ and let $T$ $=\bar{S}^{-1} \bar{R}$. Since ${ }^{*}\left(P /{ }^{*} P\right)=0,\left(P /{ }^{*} P\right) \cap \bar{S}=0$. Thus ht $\left(P /{ }^{*} P\right)=$ ht $\left(P /{ }^{*} P T\right)$. By Lemma 1 b) $T$ is simple and by Theorem $3, T$ is regular of dimension at most $\operatorname{dim} G$. Since $\operatorname{dim} T \leqq \operatorname{dim} G$, ht $\left(P /{ }^{*} P\right) \leqq \operatorname{dim} G$, and parts a) and b) follow from Corollary 6. Next, we let $Q=P /{ }^{*} P T$. Since $T$ is regular, ht $(Q)=h t\left(Q T_{Q}\right)=n$, where $n$ is the minimal number of generators of $Q T_{Q}$. But $T_{Q}=R_{P} /{ }^{*} P R_{P}$ and $Q T_{Q}=P R_{P} /{ }^{*} P R_{P}$ so c) follows.

We next turn to calculations of homological dimension.

Definition. Let $R$ be a *ring and $M$ a *module. The *projective dimension of $M$ is the least integer $n$ such that $\operatorname{Ext}_{R}^{i}(M, N)=0$ for all $i>n$ and all 
${ }^{*}$ modules $N$ and is denoted ${ }^{*} \operatorname{pd}_{R} M$. The ${ }^{*}$ injective dimension of $M$ is the least integer $n$ such that $\operatorname{Ext}_{R}^{i}(N, M)=0$ for all $i>n$ and all ${ }^{*}$ modules $N$ and is denoted $* \operatorname{id}_{R} M$. The global $*$ dimension of $R$ is the least integer $n$ such that $\operatorname{Ext}_{R}^{i}(P, Q)=0$ for all $i>n$ and all ${ }^{*}$ modules $P$ and $Q$, and is denoted ${ }^{*} \mathrm{gl} \operatorname{dim} R$.

We have the obvious inequalities ${ }^{*} \mathrm{pd}_{R} M \leqq \mathrm{pd}_{R} M, * \mathrm{id}_{R} M \leqq \mathrm{id}_{R} M$ and ${ }^{*} \mathrm{gl} \operatorname{dim} R \leqq \mathrm{gldim} R$. We next establish upper bounds for the relevant dimensions.

Theorem 8. Let $R$ be $a{ }^{*}$ ring and $M a{ }^{*}$ module. Then ${ }^{*} \operatorname{pd}_{R} M=\operatorname{pd}_{R} M$.

Proof. We observe that if $V$ is a rational $G$-module, then $R \otimes_{k} V$ is an $R$ projective *module. If $M$ is a *module, then we can regard $M$ as a rational $G$ module, and then the natural surjection $R \otimes_{k} M \rightarrow M$ is a ${ }^{*}$ homomorphism of *modules. By standard dimension shift arguments, it thus suffices to prove the theorem in case ${ }^{*} \operatorname{pd}_{R} M=0$. Let $K$ be the kernel of $\otimes_{k} M \rightarrow M$. Then in the exact sequence

$$
\operatorname{Hom}_{R}\left(M, R \otimes_{k} M\right) \rightarrow \operatorname{Hom}_{R}(M, M) \rightarrow \operatorname{Ext}_{R}^{1}(M, K),
$$

the last term is zero since $K$ is a ${ }^{*}$ module and ${ }^{*} \operatorname{pd}_{R} M=0$, so $M$ is a direct summand of $R \otimes_{k} M$ and hence is projective so $\operatorname{pd}_{R} M=0$.

Theorem 9. Let $R$ be $a{ }^{*}$ ring. Then $* \mathrm{gl} \operatorname{dim} R \leqq \operatorname{gl} \operatorname{dim} R \leqq * \operatorname{gl} \operatorname{dim} R+\operatorname{dim} G$.

Proof. We recall that

$$
\operatorname{gl} \operatorname{dim} R=\sup \left\{\operatorname{pd}_{R}(R / M) \mid M \text { a maximal ideal }\right\} .
$$

Let $M$ be a maximal ideal of $R$, let $S$ be the set of semi-invariants in $R /{ }^{*} M$, and let $T=S^{-1}(R / * M)$. Then $R / M$ can be regarded as a $T$-module, and hence $\operatorname{pd}_{R}(R / M) \leqq \operatorname{pd}_{T}(R / M)+\operatorname{pd}_{R}(T)$. By Lemma 1 and Theorem $3, T$ is regular of dimension at most $\operatorname{dim} G$, so $\operatorname{pd}_{T}(R / M) \leqq \operatorname{dim} G$, and since $T$ is a *module, $\operatorname{pd}_{R}(T)={ }^{*} \operatorname{pd}_{R}(T)$ by Theorem 8 . Thus

$$
\operatorname{pd}_{R}(R / M) \leqq \operatorname{dim} G+{ }^{*} \operatorname{pd}_{R}(T) \leqq \operatorname{dim} G+{ }^{*} \operatorname{gl} \operatorname{dim} G,
$$

and the upper bound obtains. The lower bound was previously noted.

To compute injective dimension, we need a result on the Bass numbers defined in $[1$, p. 11$]$.

Proposition 10. Let $R$ be a ${ }^{*}$ ring, $M$ an $R$-module, and $P$ a prime ideal of $R$. Suppose $\mu_{i}\left({ }^{*} P, M\right)=0$ for $i>r$. Then $\mu_{i}(P, M)=0$ for $i>r+\operatorname{dim} G$. 
Proof. By assumption, $\operatorname{Ext}_{R}^{q}\left(R /{ }^{*} P, M\right)=0$ for $q<r$. Let $T=R_{P} /{ }^{*} P R_{P}$. Then $\operatorname{Ext}_{R_{P}}^{q}\left(T, M_{P}\right)=0$ if $q>r$. If $S$ is the set of semi-invariants in $R /{ }^{*} P, S \cap P /{ }^{*} P$ $=\varnothing$ so $T$ is a localization of $S^{-1}\left(R /{ }^{*} P\right)$, and hence $T$ is regular of dimension at most $\operatorname{dim} G$ by Lemma 2 . Since $\mu_{i}(P, M)=\mu_{i}\left(P R_{P}, M_{P}\right)$, we may replace $R$ by $R_{P}$, and extend $P,{ }^{*} P$, and $M$ to $R_{P}$. By [2, p. 348] there is a spectral sequence $\operatorname{Ext}_{T}^{p}\left(R / P, \operatorname{Ext}_{R}^{q}(T, M) \Rightarrow \operatorname{Ext}_{R}^{n}(R / P, M)\right)$. Now $\operatorname{Ext}_{T}^{p}(\cdot, \cdot)=0$ for $p>\operatorname{dim} G$ and $\operatorname{Ext}_{R}^{q}(T, M)=0$ for $q>r$, so in the spectral sequence $E_{2}^{p, q}=0$ for $p+q>$ $r+\operatorname{dim} G$. Thus $\operatorname{Ext}_{R}^{n}(R / P, M)=0$ for $n>r+\operatorname{dim} G$, so $\mu_{i}(P, M)=0$ for $i>$ $r+\operatorname{dim} G$.

Corollary 11. Let $R$ be $a{ }^{*}$ ring and $M a{ }^{*}$ module. Then ${ }^{*} \operatorname{id}_{R}(M) \leqq \operatorname{id}_{R}(M)$ $\leqq * \mathrm{id}_{R}(M)+\operatorname{dim} G$.

Proof. The first inequality is clear. For the second, we need to show that $\mu_{i}(P, M)=0$ for all $i \geqq{ }^{*} \mathrm{id}_{R}(M)+\operatorname{dim} G$ for every prime ideal $P$ of $R$. By assumption, $\mu_{i}\left({ }^{*} P, M\right)=0$ for $i>* \mathrm{id}_{R}(M)$, so the result follows from Proposition 10.

Corollary 12. Let $R$ be $a{ }^{*}$ ring. Suppose $R_{P}$ is regular for every prime *ideal $P$ of $R$. Then $R$ is regular.

Proof. Let $M$ be an $R$ module, $P$ a prime ideal of $R$, and $Q \subseteq P$ a prime. Then by hypothesis $\mu_{i}\left({ }^{*} Q, M\right)=0$ for $i>$ ht $\left({ }^{*} Q\right)$. By Proposition $10, \mu_{i}(Q, M)$ $=0$ for $i>\operatorname{ht}\left({ }^{*} Q\right)+\operatorname{dim} G$, so by Theorem $7, \mu_{i}(Q, M)=0$ for $i>\operatorname{ht}(Q)$. Thus $\mu_{i}(Q, M)=0$ for $i>h t(P)$. It follows that $\operatorname{id}_{R_{P}} M_{P}$ is finite, so $R_{P}$ is regular.

Corollary 13. Let $R$ be a ${ }^{*}$ ring. Suppose $R_{P}$ is Gorenstein for every prime *ideal $P$ of $R$. Then $R$ is Gorenstein.

Proof. The argument of Corollary 12 shows that for any prime $P$ of $R$, $\mathrm{id}_{R_{P}} R_{P}$ is finite, so $R$ is Gorenstein.

To establish the analogue of Corollaries 12 and 13 for the Cohen-Macaulay property, we need a stronger version of Proposition 10, which holds when the module $M$ of that proposition is a *module. This, in turn, requires the following preliminary results.

Lemma 14. Let $R$ be a *simple *ring. Then every finitely generated *module is free as an $R$-module.

Proof. Since every finitely-generated *module has a composition series in 
which the factors are cyclic and generated by semi-invariants, it suffices to prove the lemma for modules of this type. Thus let $M$ be a *module generated by the semi-invariant $m$ of weight $\alpha$. Let $R(\alpha)$ be $R$, as an $R$-module, but with $G$ action $g \cdot r=\alpha(g) g(r)$. Then $R(\alpha)$ is a *module, and $R(\alpha) \rightarrow M$ by $r \mapsto r m$ is a surjective *homomorphism. If $R(\alpha)$ is *simple, then $M$ is $R$-free. But $R(\alpha) \otimes_{R} R\left(\alpha^{-1}\right) \rightarrow R$ by $a \otimes b \mapsto a b$ is an *isomorphism, and it follows that $R(\alpha)$ is * simple since $R$ is.

Lemma 15. Let $A$ be a commutative ring, $I$ an ideal of $A$, and $M$ an $A$-module such that $\operatorname{Ext}_{A}^{i}(A / I, M)$ is a free $A / I$-module for all $i$, and $\operatorname{Ext}_{A}^{i}(A / I, M)=0$ for $i<d$. Let $x$ be a non-zero divisor in $A / I$, and let $I=I+A x$. Then $\operatorname{Ext}_{A}^{i}\left(A / I^{\prime}, M\right)$ is a free $A / I^{\prime}$-module for all $i$ and $\operatorname{Ext}_{A}^{i}\left(A / I^{\prime}, M\right)=0$ for $i<d$.

Proof. The exact sequence $0 \rightarrow A / I \stackrel{x}{\rightarrow} A / I \rightarrow A / I^{\prime} \rightarrow 0$ yields the long exact sequence

$$
\begin{aligned}
\operatorname{Ext}_{A}^{i}(A / I, M) \rightarrow \operatorname{Ext}_{A}^{i}(A / I, M) & \rightarrow \operatorname{Ext}_{A}^{i}\left(A / I^{\prime}, M\right) \\
& \rightarrow \operatorname{Ext}_{A}^{i+1}(A / I, M) \rightarrow \operatorname{Ext}_{A}^{i+1}(A / I, M),
\end{aligned}
$$

where the first and last maps are multiplication by $x$. Since $\operatorname{Ext}_{A}^{i}(A / I, M)$ and $\operatorname{Ext}_{A}^{i+1}(A / I, M)$ are $A / I$-free, we have a short exact sequence $0 \rightarrow \operatorname{Ext}_{A}^{i}(A / I, M)$ $\stackrel{x}{\rightarrow} \operatorname{Ext}_{A}^{i}(A / I, M) \rightarrow \operatorname{Ext}_{A}^{i}(A / I, M) \rightarrow 0$ for each $i \geqq 0$. This shows that $\operatorname{Ext}_{A}^{i}\left(A / I^{\prime}, M\right)$ has the asserted properties.

TheOREM 16. Let $R$ be a *ring, $M$ a finitely generated ${ }^{*}$ module, and $P$ a prime ideal of $R$. Let $s=\mathrm{ht}(P)-\mathrm{ht}\left({ }^{*} P\right)$. Suppose $\mu_{i}\left({ }^{*} P, M\right)=0$ for $i<r$. Then $\mu_{i}(P, M)$ $=0$ for $i<r+s$.

Proof. Let $S$ be the set of semi-invariants in $R /{ }^{*} P$ and let $B=S^{-1}\left(R /{ }^{*} P\right)$. Then $B$ is *simple, and $\operatorname{Ext}_{R}^{q}(B, M)$ is a $B-{ }^{*}$ module, so $\operatorname{Ext}_{R}^{q}(B, M)$ is a free $B$ module by Lemma 14, and hence remains free when we further localize at $P$. Replace $R$ by $R_{P}$ and extend $P,{ }^{*} P$, and $M$ to $R_{P}$. Let $T=R /{ }^{*} P$. Then $\operatorname{Ext}_{R}(T, M)$ is a free $T$-module and, as in Proposition 10, we have a spectral sequence $\operatorname{Ext}_{T}^{p}\left(R / P, \operatorname{Ext}_{R}^{q}(T, M)\right) \Rightarrow \operatorname{Ext}_{R}^{n}(R / P, M)$. Since $T$ is regular, $\operatorname{Ext}_{T}^{p}(R / P, T)=0$ for $p \neq \operatorname{dim}(T)$ by [1, Prop. 3.6, p. 14]. By Corollary 6, $\operatorname{dim}(T)=\mathrm{ht}\left(P /{ }^{*} P\right)=s$. Thus the above spectral sequence collapses and we have $\operatorname{Ext}_{T}^{s}\left(R / P, \operatorname{Ext}_{R}^{q}(T, M)\right)=\operatorname{Ext}_{R}^{s+q}(R / P, M)$. Thus $\mu_{q}\left({ }^{*} P, M\right)=0 \quad$ implies $\mu_{s+q}(P, M)=0$ for all $q$. In particular, if $\mu_{i}\left({ }^{*} P, M\right)=0$ for $i<r, \mu_{i}(P, M)=0$ for $s \leqq i<r+s$. Since $T$ is regular local, $P /{ }^{*} P$ is generated by a $T$-sequence $x_{1}, \ldots, x_{s}$. Let $I_{j}={ }^{*} P+R x_{1}+\ldots+R x_{j}$, so $I_{0}={ }^{*} P$ and $I_{s}=P$. Now $\operatorname{Ext}_{R}^{i}\left(R / I_{0}, M\right)=\operatorname{Ext}_{R}^{i}(T, M)$ is a free $R / I_{0}$-module for all $i$, and $\operatorname{Ext}_{R}^{i}\left(R / I_{0}, M\right)$ 
$=0$ for $i<r$ by hypothesis. By induction and Lemma 15, $\operatorname{Ext}_{R}^{i}\left(R / I_{s}, M\right)$ $=\operatorname{Ext}_{R}^{i}(R / P, M)=0$ for $i<s$, so $\mu_{i}(P, M)=0$ for $i<s$, also.

Corollary 17. Let $R$ be $a *$ ring, and suppose $R_{P}$ is Cohen-Macaulay for every prime *ideal $P$ of $R$. Then $R$ is Cohen-Macaulay.

Proof. Let $P$ be a prime ideal of $R$, and let $Q={ }^{*} P$. By assumption, $R_{Q}$ is Cohen-Macaulay, so by $\left[1,3.7\right.$, p. 14], $\mu_{i}(Q, R)=0$ for all $i<\mathrm{ht}(Q)$. By Theorem $16, \mu_{i}(P, R)=0$ for $i<\mathrm{ht}(Q)+(\mathrm{ht}(P)-\mathrm{ht}(Q))=\mathrm{ht}(P)$; by $[1,3.7$, p. 14] again, $R_{P}$ is Cohen-Macaulay. This holds for all $P$, so $R$ is CohenMacaulay.

\section{REFERENCES}

1. H. Bass, On the ubiquity of Gorenstein rings, Math. Z. 82 (1963), 8-23.

2. H. Cartan and S. Eilenberg, Homological Algebra (Princeton Mathematical Series 19), Princeton University Press, Princeton, New Jersey, 1956.

3. R. Fossum and H. B. Foxby, The category of graded modules, Math. Scand. 35 (1974), 288-300.

4. I. Kaplansky, Commutative Rings, Allyn and Bacon, Boston, 1970.

5. H. Matsumura, Commutative Algebra, W. A. Benjamin, Inc., New York, 1970.

6. M. Rosenlicht, A remark on quotient spaces, An. Acad. Brasil Ci. 35 (1962), 487-489.

UNIVERSITY OF OKLAHOMA

601 ELM, RM. 421

NORMAN, OK 73019 USA 